\title{
An Analysis of V-blast Detection Schemes for MIMO Wireless Communications
}

\author{
Shankar.P, Dr. S.A Hariprasad \\ Department of Electronics and Communication, RV College of Engineering, Bangalore 560059, Karnataka \\ India
}

\begin{abstract}
Vertical Bell Laboratories Layered Space time (V-BLAST) is a Multiple input Multiple output $(M I M O)$ wireless communication system that uses multiple antenna elements at transmit and receive to offer high spectral efficiency and increased capacity in a rich multipath environment without increasing the use of the spectrum. Whereas the detection schemes employed in such systems are computationally expensive as the number of transmitting and receiving antennas increases. Reduction in computational cost is essential for time critical application of high speed packet transfer using MIMO for 3GPP and 3GPP-2 standards. This paper compares the performance between various detection scheme which include the conventional detection, An efficient square root algorithm, An improved square root algorithm and An improved square root algorithm based on cholesky factorization when subjected to multi-media application with $4 \times 4$ and $12 x 12$ array for varying SNR. Performance parameters considered include bit error rate (BER), symbol error rate (SER), peak signal-to-noise ratio (PSNR), number of number of floating point operations (FLOPS) and Time required for detection. Among the Modulation scheme optimum performance is achieved with M-QAM, whereas among the detection schemes nonlinear detection schemes with interference cancellation (IC) with MMSE performed better than ZF schemes in terms of BER, SER PSNR but at the cost of increase in FLOPS. In an attempt to reduce computational complexity the number of FLOPS required for detection with an improved square root algorithm based on efficient inverse cholesky factorization with MMSE for 12 transmitting and 12 receiving antennas is $0.3 \times 10^{6}$, a reduction of $0.7 \times 10^{6}, 0.9 \times 10^{6}, 1.5 \times 10^{6}$ and $1.7 \times 10^{6}$ flops is achieved when compared to improved square-root algorithm, the efficient square root algorithm and the conventional detection scheme employing Zero forcing (ZF) and MMSE filter respectively. This algorithm is faster than the existing efficient V-BLAST algorithms.
\end{abstract}

Index Terms-Multiple-input-multiple-output (MIMO) systems, Bell Laboratories Layered Space time (BLAST), vertical BLAST (V-BLAST), Zero forcing (ZF), Bit error rate (BER), Symbol error rate (SER), peak signal-to-noise ratio (PSNR), Floating point operations (FLOPS).

\section{INTRODUCTION}

Digital communication using Multiple-Input-Multiple-Output (MIMO) wireless systems, characterized by multiple antenna elements at the transmitter and receiver, have demonstrated the potential for increased capacity in rich multipath environments [1]-[4]. Such systems operate by exploiting the spatial properties of the multipath channel, thereby offering a new dimension which can be used to enhance communication performance. Bell Labs Layered Space-Time architecture (BLAST) [5], including the relative simple vertical BLAST (V-BLAST) [6], is such a system that maximizes the data rate by transmitting independent data streams simultaneously from multiple antennas. V-BLAST often adopts the ordered successive interference cancellation (OSIC) detector [6], which detects the data streams iteratively with the optimal ordering. In each iteration the data stream with the highest signal-to-noise ratio (SNR) among all undetected data streams is detected through Zero-forcing (ZF) or minimum mean square error (MMSE) filter. This is referred to as nulling and cancellation. The optimal detection order is from the strongest to the weakest signal, since this minimizes propagation of error from one step of detection to the next step. Further the effect of the detected data stream is subtracted from the received signal vector. This is referred to as interference cancellation. It turns out that the main computational bottleneck in the conventional detection algorithm is the step where the optimal ordering for the sequential estimation and detection of the transmitted signals, as well as the corresponding so called nulling vector is determined. Current implementations devote $90 \%$ of the total computational cost to this step. This high computational cost limits the scope of the application that admits inexpensive real time solutions. Moreover, when the numbers of transmitting and receiving antennas are large repeated pseudo-inverse that conventional detection algorithm requires can lead to numerical instability, thus a numerically robust and stable algorithm is required. In an attempt to reduce the computational complexity an efficient square-root [7-8] algorithm has 
been proposed. The algorithm is numerically stable since it is division free and uses only Orthogonal transformations such as Householders transformation or sequence of Givens Rotation[9][10]. To further reduce the computational cost An Improved square root

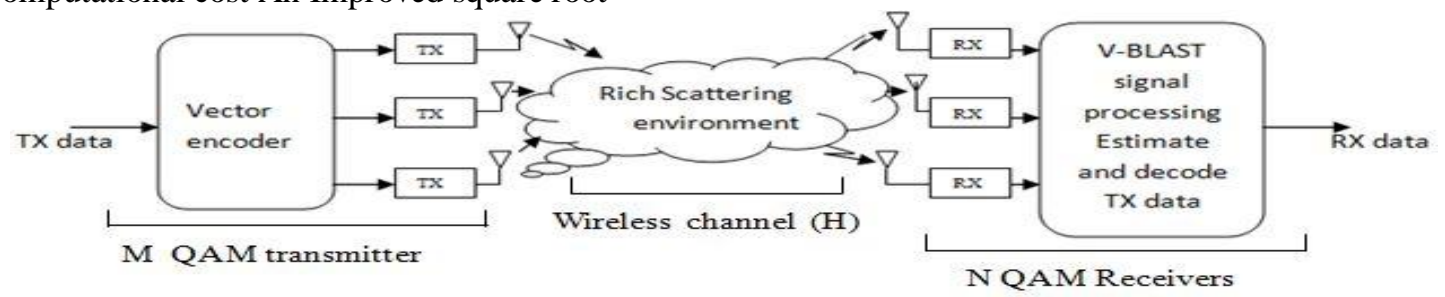

Figure 1: V-BLAST system model

algorithm has been proposed [11] which speed up's the original square root algorithm by $45 \%$ in terms of number addition and multiplication by reusing intermediate results. An Improved Square-Root Algorithm for VBLAST Based on Efficient Inverse Cholesky Factorization [12] computes a triangular square root of the estimation error of the covariance matrix using Inverse Cholesky Factorization and is then applied to An Improved square root algorithm which can offer further computational savings. The algorithm is faster than the existing efficient V-BLAST detection algorithms.

The remainder of the paper is organized as follows Section II describes the V-BLAST system model Section III introduces different V-BLAST detection schemes which include Conventional Detection Algorithm, An Efficient Square-Root Algorithm, An Improved Square Root Algorithm and An Improved Square root Algorithm based on Efficient Inverse Cholesky Factorization along with their simulation results. Finally we make conclusion in Section IV

In the following sections, $(\cdot),(\cdot)^{*}$ and $(\cdot)^{H}$ denote matrix transposition, matrix conjugate, and matrix conjugate transposition, respectively. $\mathbf{0}_{M}$ is the $M \times 1$ zero column vector, while $\mathrm{I}_{M}$ is the identity matrix of size $M$.

\section{SYSTEM MODEL}

The V-BLAST system consists of $\mathrm{M}$ transmitting and $\mathrm{N}$ receiving antennas in a rich-scattering environment illustrated in Figure. 1 where a single data stream is de-multiplexed into $M$ sub streams and each sub stream is then encoded into symbols and fed to its respective transmitter. The Transmitters 1 to $M$ operate co-channel at symbol rate 1/ $T$ symbols/sec, with synchronized symbol timing. Each transmitter is itself an ordinary QAM transmitter. The collection of transmitters comprises, in effect, a vector-valued transmitter, where components of each transmitted M-vector are symbols drawn from a QAM constellation. The power launched by each transmitter is proportional to $1 / \mathrm{M}$ so that the total radiated power is constant and independent of M.

Let the Signal vector transmitted from $\mathrm{M}$ antennas is $\mathrm{a}=\left[\mathrm{a}_{1}, \mathrm{a}_{2}, \ldots \ldots \mathrm{a}_{\mathrm{M}}\right]^{\mathrm{T}}$ with the co-variance $\mathrm{E}\left(\mathrm{aa}^{\mathrm{H}}\right)=\sigma_{v}^{2}$. Then the received vector $(r)$ is given by

$$
r=H \cdot a+w,
$$

Where $w$ is the $N \times 1$ zero-mean circular symmetric complex Gaussian (ZMCSCG) noise vector with the zero mean and the covariance $\sigma_{w}^{2} \mathrm{I}_{\mathrm{N}}$ and $\mathrm{H}=\left[\mathrm{h}_{1}, \mathrm{~h}_{2} \ldots . . \mathrm{h}_{\mathrm{M}}\right]=\left[\underline{\mathrm{h}}_{1} \underline{\mathrm{h}}_{2} \ldots \ldots \underline{\mathrm{h}}_{\mathrm{M}}\right]^{\mathrm{H}}$ is the $\mathrm{N} \times \mathrm{M}$ complex matrix. $\mathrm{h}_{\mathrm{m}}$ and $\underline{\mathrm{h}}_{\mathrm{m}}$ are the $\mathrm{m}$-th column and the $\mathrm{n}$-th row of $\mathrm{H}$, respectively.

The Linear zero-forcing (ZF) estimate of a is

$$
\hat{a}=\mathrm{H}^{+} \mathrm{r}=\left(\mathrm{H}^{\mathrm{H}} \mathrm{H}\right)^{-1} \mathrm{H}^{\mathrm{H}} \mathrm{r} \text {. }
$$

Define $\alpha=\sigma_{w}^{2} / \sigma_{v}^{2}$. The Linear minimum mean square error (MMSE) estimate of a is

$$
\hat{a}=\left(\mathrm{H}^{\mathrm{H}} \mathrm{H}+\alpha \mathrm{I}_{\mathrm{M}}\right)^{-1} \mathrm{H}^{\mathrm{H}} \mathrm{r} \text {. }
$$

Let $\mathrm{R}=\left(\mathrm{H}^{\mathrm{H}} \mathrm{H}+\alpha \mathrm{I}_{\mathrm{M}}\right)$.Then the estimation error covariance matrix [4] $\mathrm{P}$ is given by

$$
\mathrm{P}=\mathrm{R}^{-1}=\left(\mathrm{H}^{\mathrm{H}} \mathrm{H}+\alpha \mathrm{I}_{\mathrm{M}}\right)^{-1}
$$

The Ordered successive Interference Cancellation (OSIC) detection detects $M$ entries of the transmit vector ' $\mathrm{a}$ ' iteratively with the optimal ordering. In each iteration, the entry with the highest SNR among all the undetected entries is detected by a linear filter, and then its interference is cancelled from the received signal vector [5].

Suppose that the entries of ' $a$ ' are permuted such that the detected entry is $a_{M}$, the M-th entry. Then the Interference is cancelled by

$$
\mathrm{r}^{\mathrm{M}-1}=\mathrm{r}^{\mathrm{M}}-\mathrm{h}_{\mathrm{M}} \mathrm{a}_{\mathrm{M}}
$$

where $\mathrm{a}_{\mathrm{M}}$ is treated as the correctly detected entry and the initial $\mathrm{r}^{\mathrm{M}}=r$. Then the reduced order problem is

$$
\mathrm{r}^{\mathrm{M}-1}=\mathrm{h}_{\mathrm{M}-1} \mathrm{a}_{\mathrm{M}-1}+\mathrm{w}
$$

where the deflated channel matrix $\mathrm{H}_{\mathrm{M}-1}=\left[\mathrm{h}_{1}, \mathrm{~h}_{2} \ldots \ldots \mathrm{h}_{\mathrm{M}-1}\right]$ and the reduced transmit vector $\mathrm{a}_{\mathrm{M}-1}=\left[\mathrm{a} 1, \mathrm{a} 2, \ldots \mathrm{a}_{\mathrm{M}-1}\right]^{\mathrm{T}}$. The Linear estimate of $\mathrm{a}_{\mathrm{M}-\mathrm{I}}$ can be deduced from (6).The detection will proceed iteratively until all entries are detected. 


\section{V-BLAST DETECTION SCHEMES}

The V-BLAST detection schemes are summarized as follows:

\section{A. Conventional detection Scheme}

a) Compute a linear transform matrix (P) for nulling. The most common criteria for nulling are zeroforcing (7) and minimum mean square error (8) for which the corresponding linear transform matrix are

$$
\begin{aligned}
& \mathrm{P}=\mathrm{H}^{+}=\left(\mathrm{H}^{\mathrm{H}} \mathrm{H}\right)^{-1} \mathrm{H}^{\mathrm{H}} \\
& \mathrm{P}=\left(\mathrm{H}^{\mathrm{H}} \mathrm{H}+\alpha \mathrm{I}_{\mathrm{M}}\right)^{-1} \mathrm{H}^{\mathrm{H}}
\end{aligned}
$$

Where ${ }^{+}$denotes the Moore-Penrose pseudo-inverse and ${ }^{\mathrm{H}}$ denotes the Hermitian matrix.

b) Determine the optimal ordering for detection of the transmitted symbol by

\section{Iterative Detection:}

$$
\mathrm{k}=\operatorname{argmin}\left\|(\mathrm{P})_{\mathrm{j}}\right\|^{2}
$$

c) Obtain the $\mathrm{k}^{\text {th }}$ nulling vector $\mathrm{W}_{\mathrm{k}}$ by

Where $(\mathrm{P})_{\mathrm{k}}$ is the $\mathrm{k}^{\text {th }}$ row of $\mathrm{P}$

$$
\mathrm{W}_{\mathrm{k}}=(\mathrm{P})_{\mathrm{k}}
$$

d) Using nulling vector $\mathrm{W}_{\mathrm{k}}$ form decision statistic $\mathrm{y}_{\mathrm{k}}$ :

$$
\mathrm{y}_{\mathrm{k}}=\mathrm{W}_{\mathrm{k}} \mathrm{r}_{\mathrm{i}}
$$

Where $r$ is the received symbols which is a column vector

e) Slice $y_{k}$ to obtain $\hat{a}_{k}$

$$
\hat{a}_{\mathrm{k}}=\mathrm{Q}\left(\mathrm{y}_{\mathrm{k}}\right)
$$

Where $\mathrm{Q}$ (.) denotes the quantization (slicing) operation appropriate to the constellation in use

f) Interference Cancellation or the Reduced order problem: Assuming that $\hat{a}_{\mathrm{k}}=\mathrm{a}_{\mathrm{k}}$, cancel $\mathrm{a}_{\mathrm{k}}$ from the received vector $r$ resulting in modified received vector $r 1$ :

Where $(\mathrm{H})_{\mathrm{k}}$ denotes the $\mathrm{k}^{\text {th }}$ column of $\mathrm{H}$

$$
r_{i+1}=r_{i}-\hat{a}_{k}(H)_{k}
$$

g) Deflate $\mathrm{H}$ denoted by $\mathrm{H}_{\overline{\mathrm{k}}}$

$$
\mathrm{H}=\mathrm{H}_{\mathrm{k}}^{-}
$$

h) Form the linear transform matrix $(\mathrm{P})$ utilizing the deflated $\mathrm{H}$ depending upon the criteria for nulling chosen, zero-forcing (7) and minimum mean square error (8).

i) Determine the optimal ordering for detection of the transmitted symbol by

j) If $\mathrm{i}>1$, let $\mathrm{i}=\mathrm{i}-1$ and go back to step 3

$$
\mathrm{k}=\operatorname{argmin}\left\|(\mathrm{P})_{\mathrm{j}}\right\|^{2}
$$

\section{Simulation Results}

The simulation is performed using the following parameters:

TABLE I : SIMULATION PARAMETERS

\begin{tabular}{|l|c|}
\hline $\begin{array}{l}\text { Antenna Configurations } \\
\text { (Transmitting X Receiving) }\end{array}$ & $4 \times 4$ and 12X12 \\
\hline \multicolumn{1}{|c|}{ Input Image Dimension } & 384 X256 \\
\hline SNR (db) & None 25 \\
\hline Compression Applied & 4 \\
\hline Frame Size Assumed & $\begin{array}{l}\text { Rayleigh Flat Fading } \\
\text { varying randomly with } \\
\text { every frame }\end{array}$ \\
\hline Channel Characteristics & $\begin{array}{l}\text { 4,16,64,256,1024 } \\
\text { QAM,PAM,PSK }\end{array}$ \\
\hline Modulation and & \\
\hline
\end{tabular}

From Figure 2,3 (a),(b),(c),(d),(e),(f) BER, SER comparison between zero-forcing (ZF) and minimum mean square error (MMSE) (In the ascending order 1st (Lower most) black line, 2nd black line, 3rd black line, 4th black line, 5th (upper most) black line indicate BER observed employing MMSE detection scheme with 4QAM,PAM,PSK modulation, 16-QAM, PAM, PSK modulation, 64-QAM, PAM ,PSK modulation, 256-QAM, PAM ,PSK modulation and 1024-QAM, PAM ,PSK modulation respectively. The 1st (Lower most) blue line, 2nd blue line, 3rd blue line, 4th blue line, 5th (upper most) blue line indicate BER observed employing ZF with 4-QAM, PAM,PSK modulation,16-QAM, PAM,PSK modulation, 64-QAM, PAM,PSK modulation,256-QAM, PAM ,PSK modulation and 1024-QAM, PAM, PSK modulation respectively).

From Figure 2,3 (a),(b),(c),(d),(e),(f) BER, SER comparison between zero-forcing (ZF) and minimum mean square error (MMSE) we observe the Bit Error Rate and Symbol Error Rate obtained for MMSE is lower 
than that of $\mathrm{ZF}$ due to the regularization $\left(\alpha \mathrm{I}_{\mathrm{M}}\right)$ introduced in MMSE, which introduces a bias that leads to a much more reliable result than ZF when the matrix is ill-conditioned and when the estimation of the channel is noisy. From figures we also observe MMSE outperforms ZF only when the Modulation scheme employed has lower constellation i.e. at lower data rates (4, 16, 64, 256 QAM,PAM,PSK), but at higher constellation i.e. at 1024 QAM,PAM,PSK Modulation scheme the BER obtained using MMSE and ZF are similar which is independent for given antenna configuration. Optimum BER and SER can also be achieved by increasing the number of transmitting and receiving antennas [7]. The gaps observed in the graph indicate a BER of zero i.e. the transmitted image was received without any errors.

In Figure 4(a),(b),(c),(d),(e),(f) In the ascending order, $1^{\text {st }}$ (Lower most) black line, $2^{\text {nd }}$ black line, $3^{\text {rd }}$ black line, $4^{\text {th }}$ black line, $5^{\text {th }}$ (upper most) black line indicate PSNR observed employing MMSE detection scheme with 1024-QAM, PAM, PSK modulation, with 256-QAM, PAM, PSK modulation, with 64-QAM, PAM, PSK modulation, with 16-QAM, PAM, PSK modulation and with 4-QAM, PAM, PSK modulation respectively. The $1^{\text {st }}$ (Lower most) blue line, $2^{\text {nd }}$ blue line, $3^{\text {rd }}$ blue line, $4^{\text {th }}$ blue line, $5^{\text {th }}$ (upper most) blue line indicate PSNR observed employing ZF with 1024-QAM, PAM, PSK modulation, with 256 -QAM, PAM, PSK modulation, with 64-QAM, PAM, PSK modulation, with 16-QAM, PAM, PSK modulation , and with 4 -QAM , PAM, PSK modulation respectively

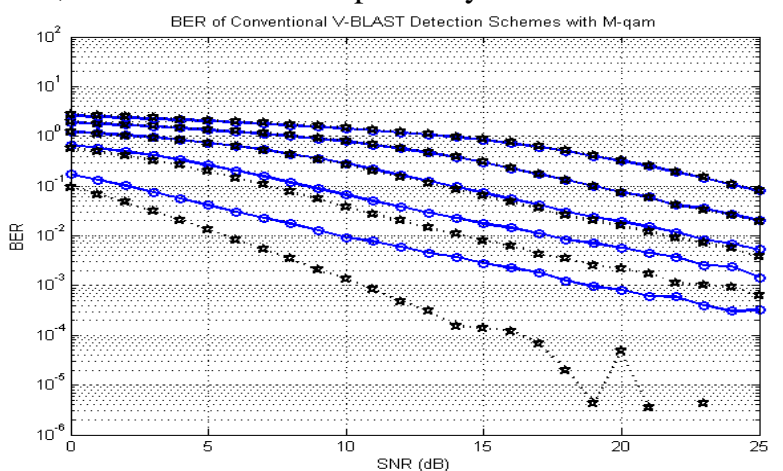

(a)

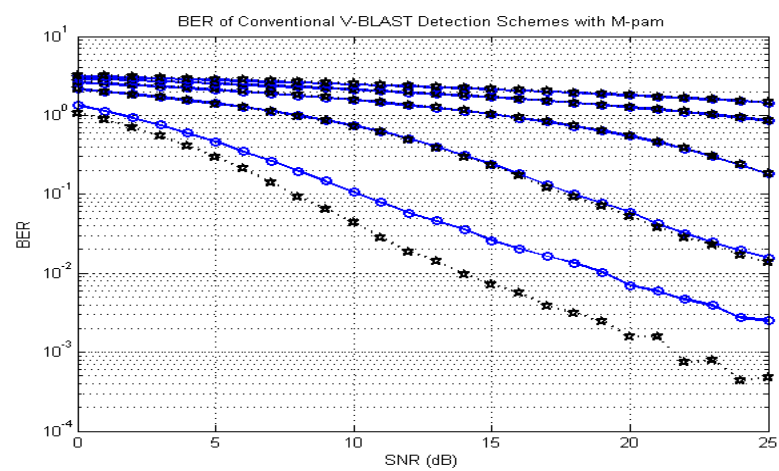

(b)

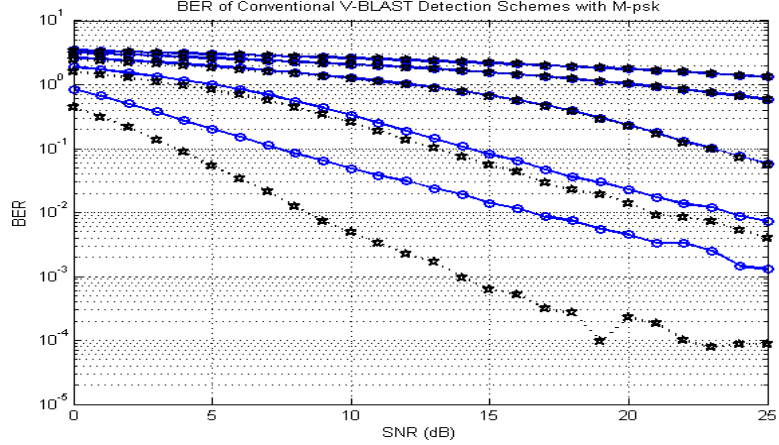

(e)

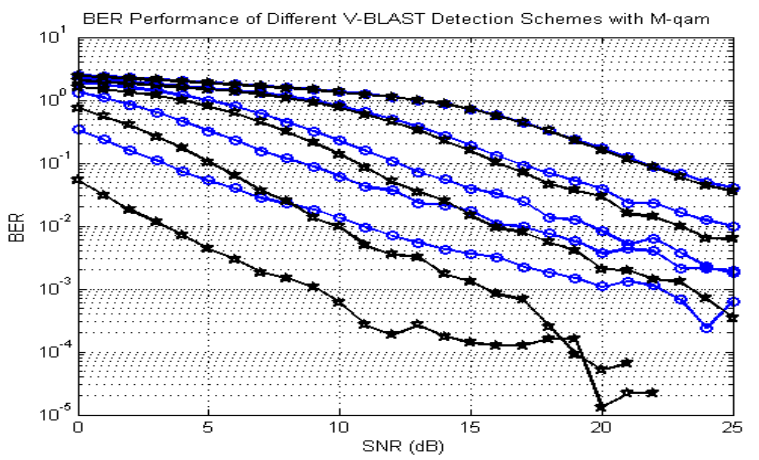

(b)

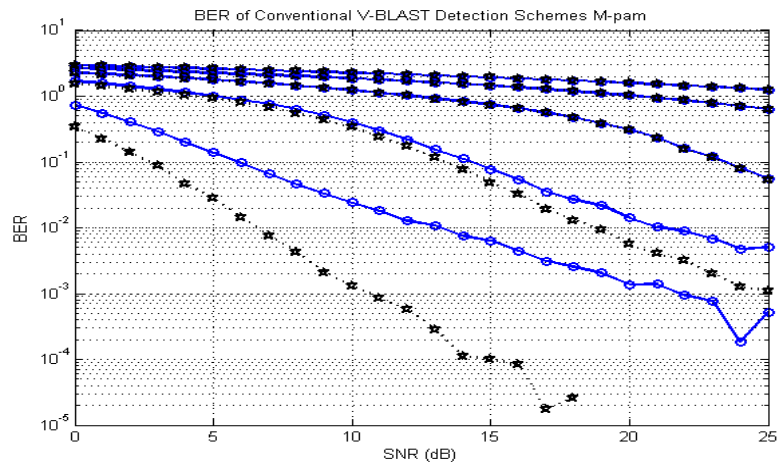

(d)

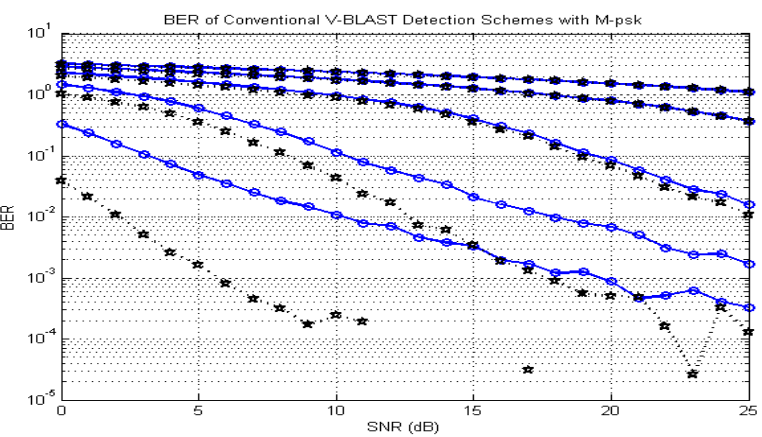

(f)

Figure 2: BER comparison between Zero-Forcing (ZF) (blue) and Minimum Mean Square Error (MMSE) (black) a) BER observed for a 4x4 antenna configuration with M-qam [13] b) BER observed for a $12 \times 12$ antenna configuration with M-qam c) BER observed for a 4x4 antenna configuration with M-pam [15] d) BER observed for a $12 \times 12$ antenna configuration with M-pam e) BER observed for a $4 \times 4$ antenna configuration with M-psk [15] f) BER observed for a 12x12 antenna configuration with M-psk. 


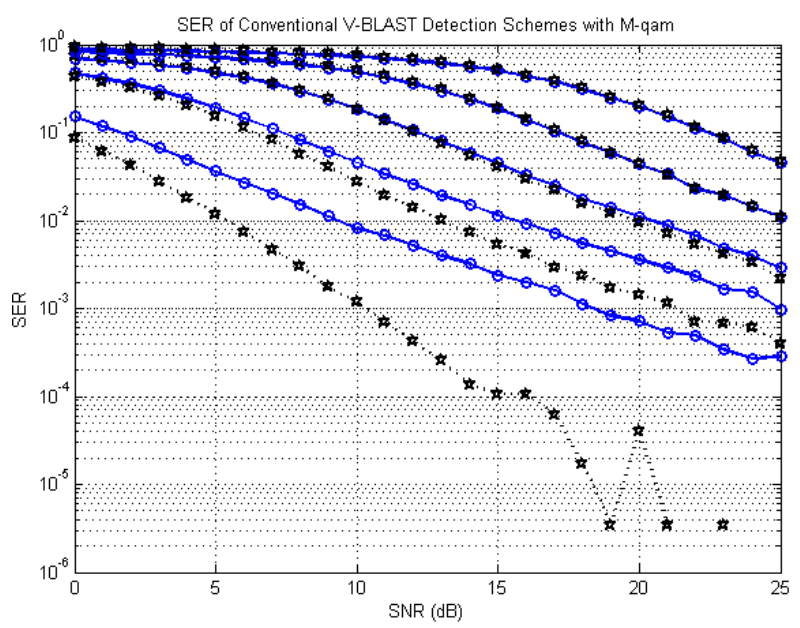

(a)

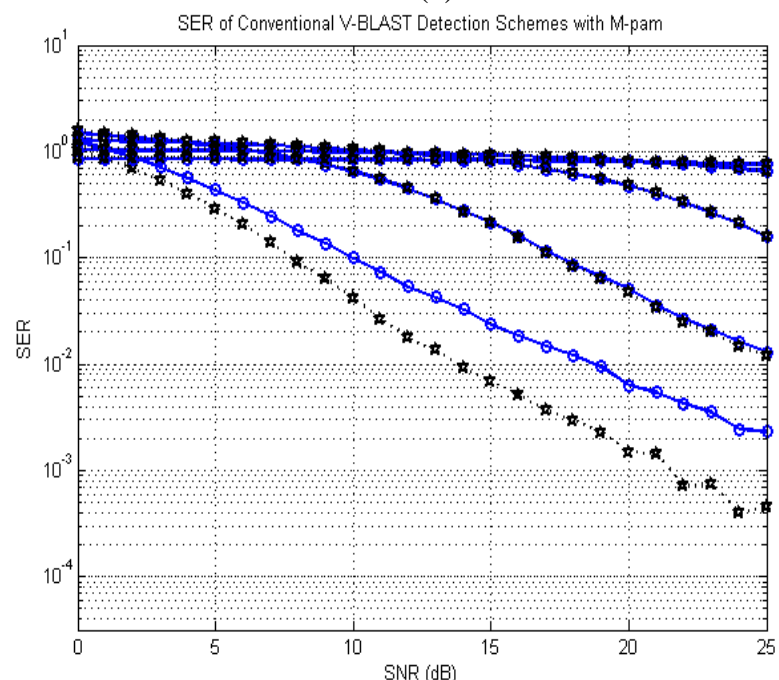

(c)

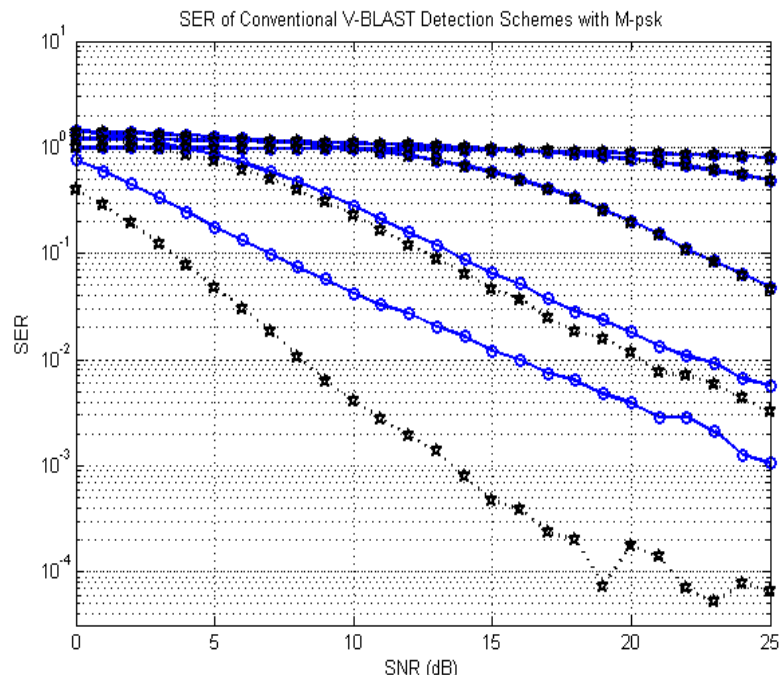

(e)

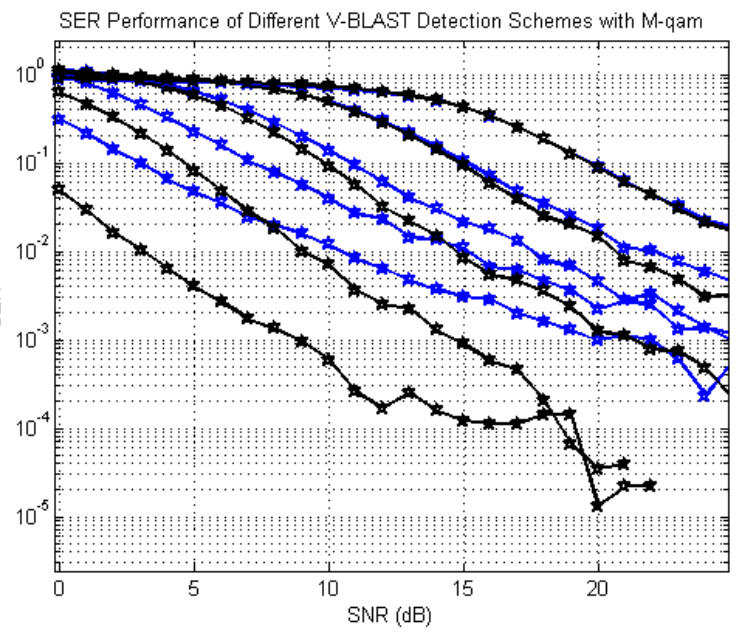

(b)

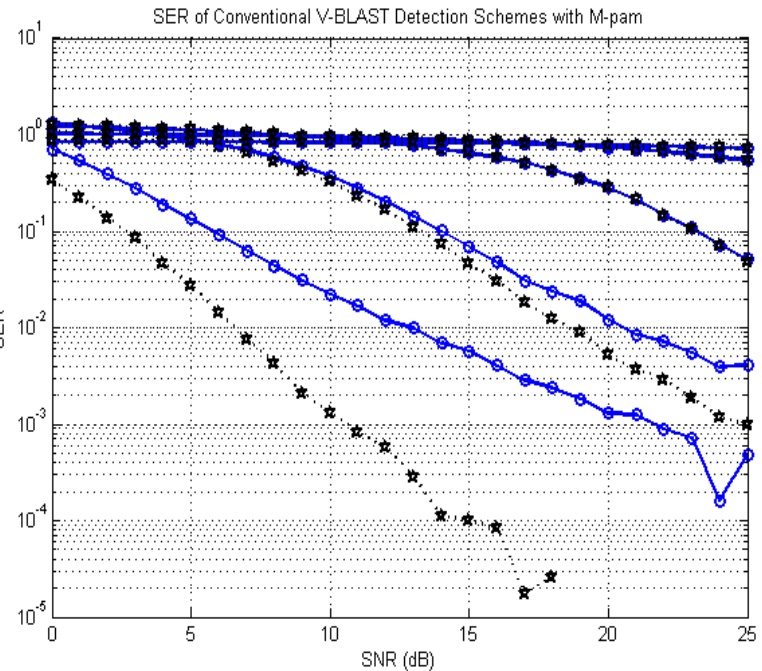

(d)

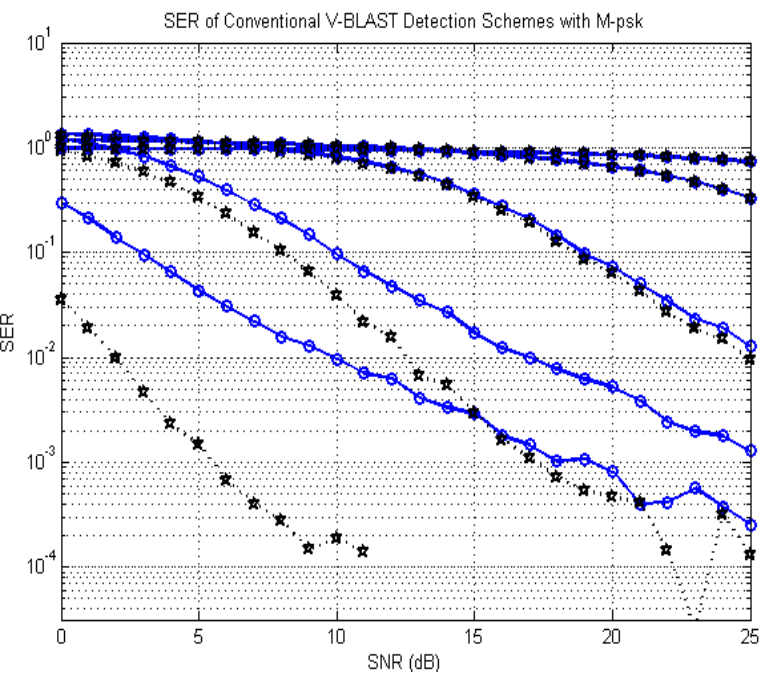

(f)

Figure 3: SER comparison between Zero-Forcing (ZF) (blue) and Minimum Mean Square Error (MMSE) (black) a) SER observed for a 4x4 antenna configuration with M-qam [13] b) SER observed for a 12x12 antenna configuration with M-qam c) SER observed for a $4 \times 4$ antenna configuration with M-pam d) SER

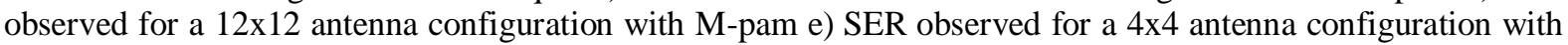
M-psk f) SER observed for a 12x12 antenna configuration with M-psk. 


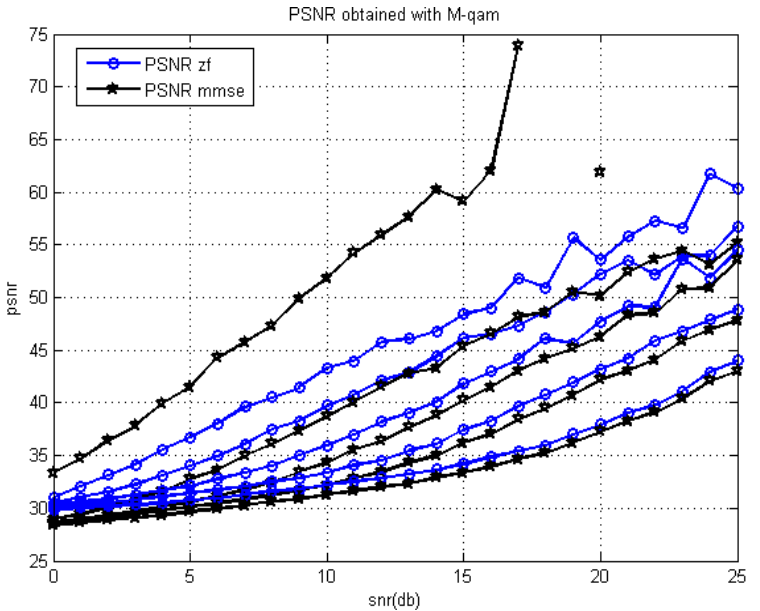

(a)

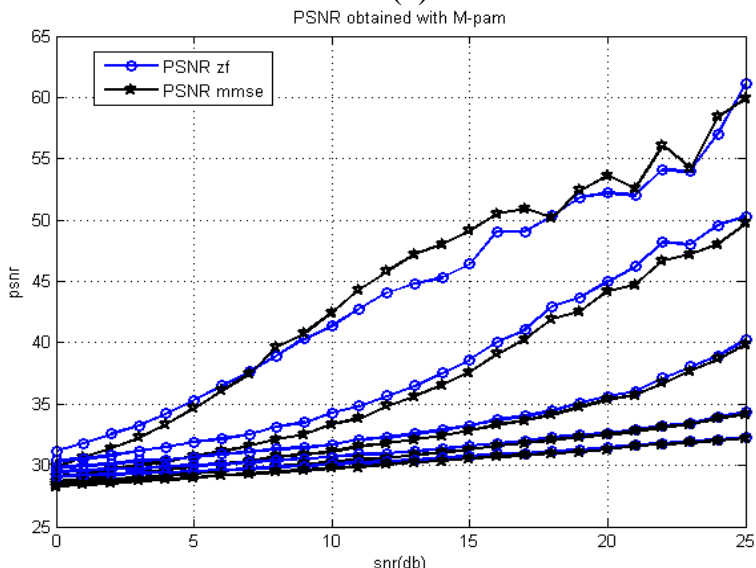

(c)

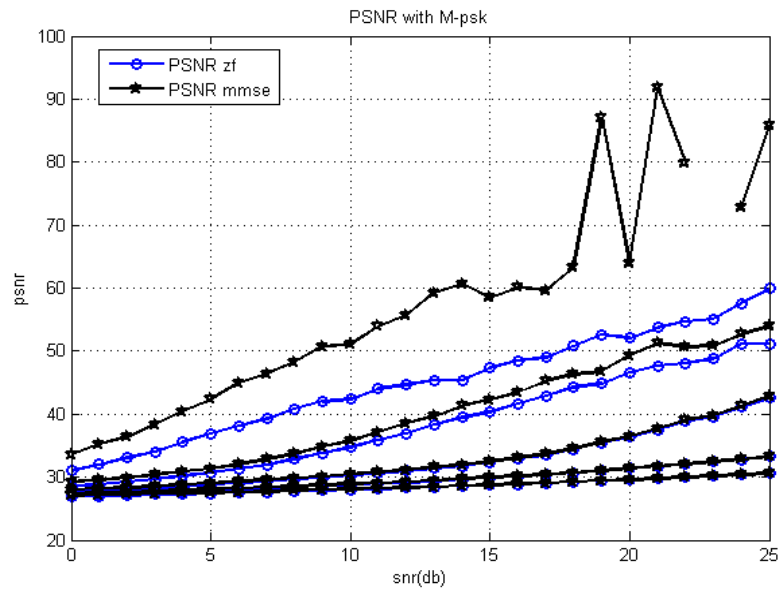

(e)

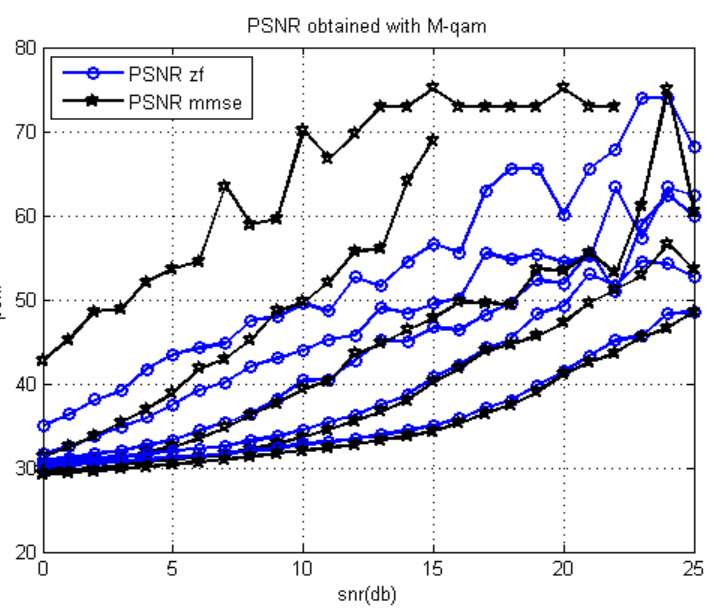

(b)

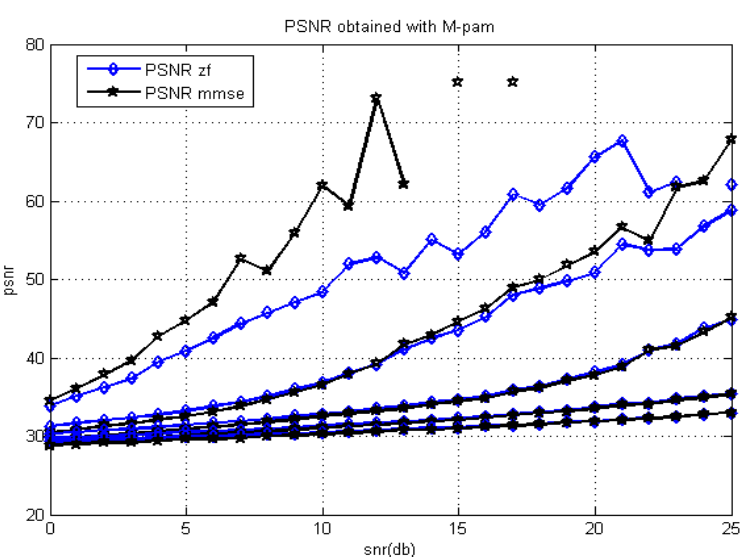

(d)

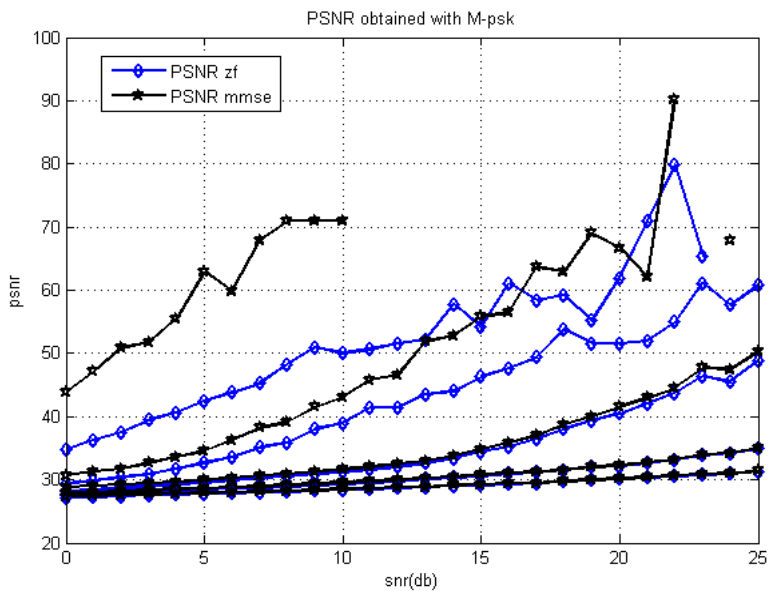

(f)

Figure 4: PSNR Comparison between the Reconstructed Output and the Original Image Transmitted For ZeroForcing (ZF) (blue) and Minimum Mean Square Error (MMSE) (black) (a) PSNR observed for a 4x4 antenna configuration with M-qam [13] (b) PSNR observed for a 12x12 antenna configuration with M-qam (c) PSNR observed for a $4 \times 4$ antenna configuration with M-pam [15] (d) PSNR observed for a 12x12 antenna configuration with M-pam (a) PSNR observed for a $4 \times 4$ antenna configuration with M-psk [15] (a) PSNR observed for a $12 \times 12$ antenna configuration with M-psk 


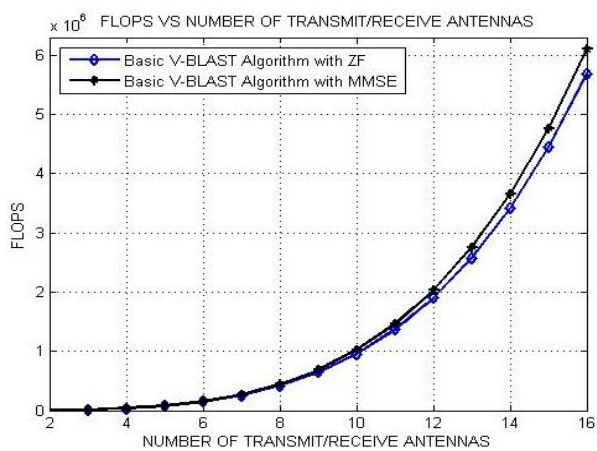

(a)

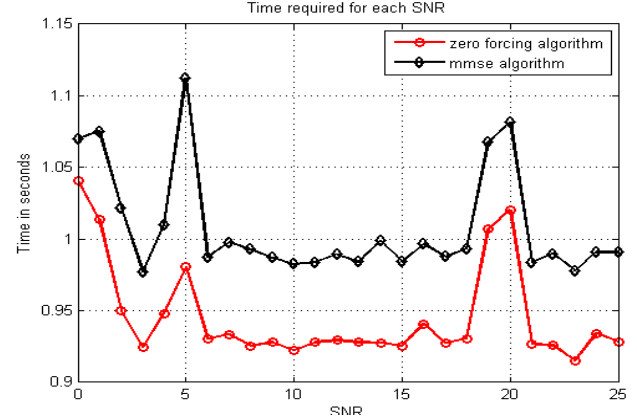

(c)

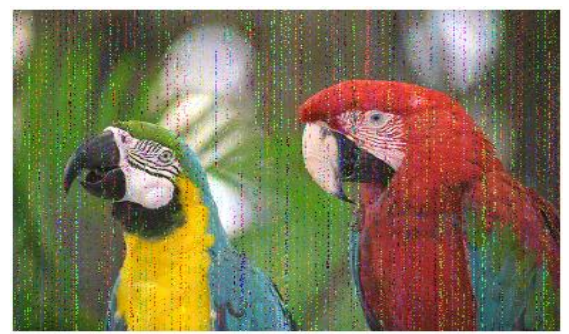

(e)

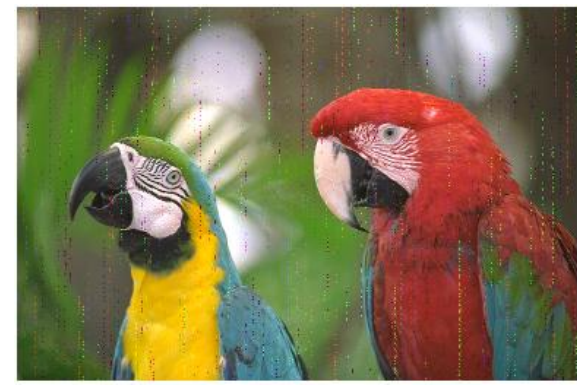

(g)

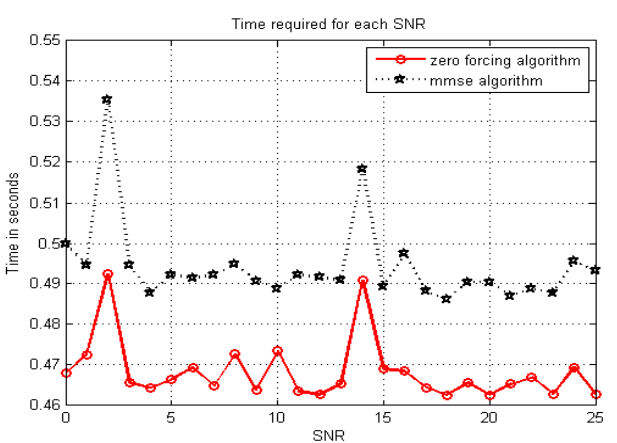

(b)

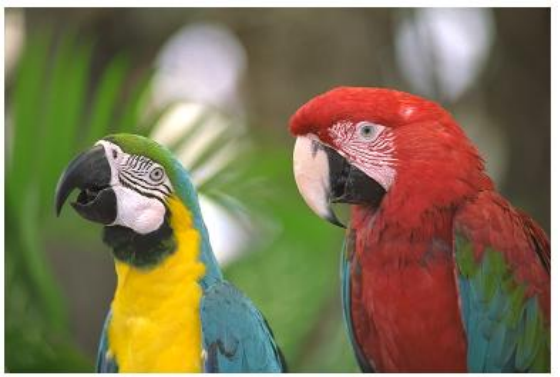

(d)

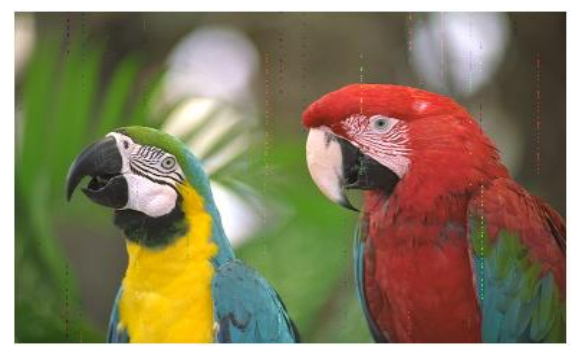

(f)

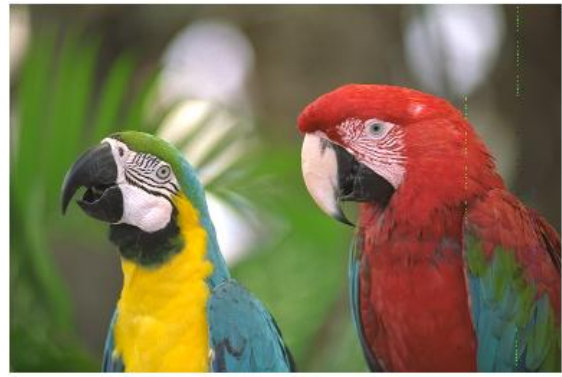

(h)

Figure 5: (a) TOTAL FLOPS required for ZF and MMSE [13] (b) Time required for 4x4 antenna configuration [13] (c) Time required for 12x12 antenna configuration (d) Original Transmitted Image (e) Reconstructed Output Image of ZF algorithm at SNR=0 (f) Reconstructed Output Image of ZF algorithm at $\mathrm{SNR}=20$ (g) Reconstructed Output Image of MMSE algorithm at SNR=0 (h) Reconstructed Output Image of MMSE algorithm at $\mathrm{SNR}=20$.

From figure 4 we observe the difference in the quality of the image reconstructed at the receiver when compared to the original image that was transmitted. The Quality of the Image Reconstructed i.e. the PSNR is higher at lower constellation i.e. at lower data rates (4, 16, 64, 256 QAM, PAM, PSK), but at higher constellation i.e. at 1024 QAM , PAM, PSK Modulation scheme the PSNR obtained using MMSE and ZF are similar. Improvement in PSNR is also observed when the number of transmitting and receiving antennas is increased [7]. The gaps observed in the graph indicate a PSNR of infinity. 
From Figure 5(a) we observe the Number of Floating Point Operations (FLOPS) required for MMSE and ZF increases monotonically for Number of Transmit and Receiving antennas from 1 to 10, above which further increase in Number of Floating Point operations is observed for MMSE when compared to ZF. (One complex multiplication and addition requires six and two flops respectively). From figure 5(b), (c) compares the time required for detection for ZF and MMSE detection algorithms for 4x4 and 12x12 array respectively. The Time required is directly related to the Number of flops required for execution.

Figure 5:(d) is the original transmitted Image, Figure 5 (e),(f), (g),(h) are the Reconstructed Image at the receiver for SNR= 0 and 20 for MMSE and ZF algorithm. The Quality of the image is directly related to the BER observed, since the BER of MMSE outperforms ZF the quality of image obtained using MMSE is higher when compared to ZF.

\section{B. An Efficient Square-Root Algorithm}

The main computational bottleneck in the Basic BLAST detection algorithm is the "nulling and cancelation" step, where the optimal ordering for the sequential estimation and detection of the received signal is determined. An Efficient Square-Root Algorithm [8] for BLAST reduces the computational cost for the nulling and cancellation step. The algorithm is numerically stable since it is division free and uses only Orthogonal transformations such as Householders transformation or sequence of Givens Rotation[9][10] .The numerical stability of the algorithm also makes it attractive for implementation in fixed-point rather than floating-point, architectures.

\section{Initialization:}

a) Let $\mathrm{m}=\mathrm{M}$. Compute square root of $\mathrm{P}$, i.e., $\mathrm{P}^{1 / 2}$ and $\mathrm{Q}_{\alpha}$

Form the so called $(\mathrm{M}+\mathrm{N}+1) \times(\mathrm{M}+1)$ pre array

$$
\Omega_{\mathrm{i}-1}=\left[\begin{array}{cc}
1 & \mathrm{H} P_{i-1}^{1 / 2} \mathrm{i} \\
0_{\mathrm{M}} & P_{i-1}^{1 / 2} \\
-\mathrm{e}_{\mathrm{i}} & \mathrm{B}_{\mathrm{i}-1}
\end{array}\right]
$$

and propagate the pre-array $\mathrm{N}$ times:

$$
\Omega_{\mathrm{i}-1} \Delta_{\mathrm{i}}=\left[\begin{array}{cc}
\mathrm{x} & 0_{M}^{T} \\
\mathrm{x} & P_{i-1}^{172} \\
\mathrm{x} & \mathrm{B}_{\mathrm{i}}
\end{array}\right]
$$

where $e_{i}$ is an $N * 1$ vector of all-zeros except for the $i$-th entry which is unity, $P^{1 / 2}$ is the square root of an $\mathrm{M} \times \mathrm{M}$ linear transform matrix $\mathrm{P}$ for MMSE is given by

$$
\mathrm{P}=\left(\mathrm{H}^{\mathrm{H}} \mathrm{H}+\mathrm{I}_{\mathrm{M}}\right)^{-1} \mathrm{H}^{\mathrm{H}}
$$

$\mathrm{B}_{\mathrm{i}}$ is an $\mathrm{N} \times \mathrm{M}$ sub-matrix of $\Omega_{\mathrm{i}}$ and $\mathrm{B}_{\mathrm{N}}=\mathrm{Q}_{\alpha}$, “×”denotes not relevant entries at this time, and $\Delta_{\mathrm{i}}$ is any unitary transformation that block lower triangularize the pre-array ${ }^{\prime} \Omega_{\mathrm{i}}$.

\section{Iterative Detection:}

b) Find the minimum length row of $\mathrm{P}^{1 / 2}$ and permute it to be the last M-th row.

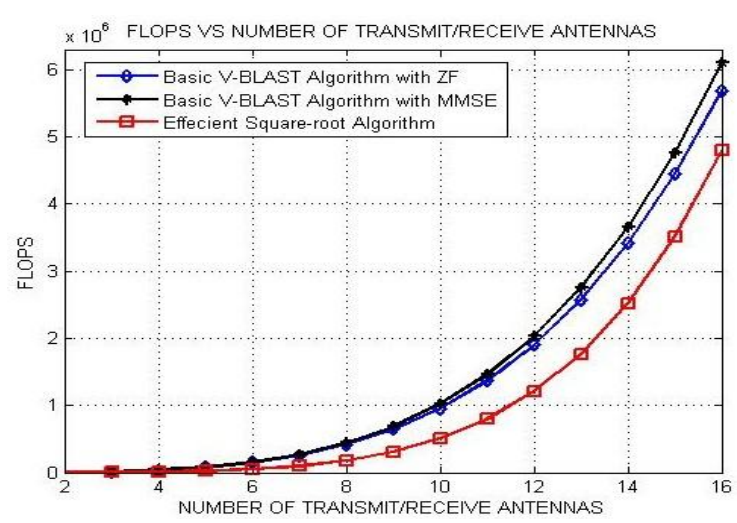

(a)

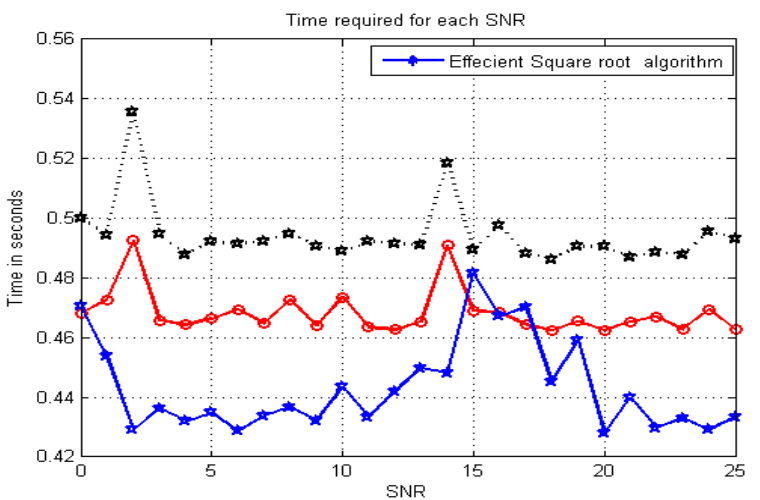

(b) 


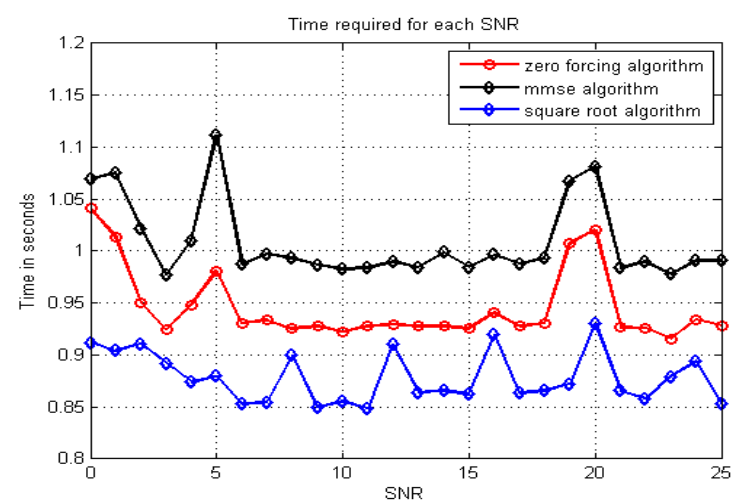

(c)

Figure 6: (a) TOTAL FLOPS required for ZF and MMSE and an efficient square root algorithm employing MMSE [13] (b) Time required for detection for $4 \times 4$ antenna configuration with efficient square root algorithm [13] (c) Time required for detection for $12 \times 12$ antenna configuration with efficient square root algorithm (Black: conventional detection with MMSE; red conventional detection with ZF; blue: efficient square root algorithm employing MMSE)

c) Find a unitary transformation $\Sigma$ such that $\mathrm{P}^{1 / 2} \Sigma$ is block Upper triangular

$$
\mathrm{P}^{1 / 2} \Sigma=\left[\begin{array}{ll}
\mathrm{P}^{(\mathrm{M}-1) / 2} & P_{M}^{(M-1) / 2} \\
0_{M-1}^{T} & P_{M}^{1 / 2}
\end{array}\right]
$$

Where $P_{M}^{(M-1) / 2}$ and $P_{M}^{1 / 2}$ denote the last (M-1) $\times 1$ sub-column and the (M,M)-th scalar entry, respectively.

d) Update $\mathrm{Q}_{\alpha}$ to $\mathrm{Q}_{\alpha} \Sigma$

e) Form the linear MMSE estimate of $a_{m}$,

Where $\mathrm{q}_{\alpha, \mathrm{M}}$ is the M-th column of $\mathrm{Q}_{\alpha}$.

$$
\widehat{\mathrm{a}}_{\mathrm{m}}=P_{M}^{1 / 2} q_{\alpha, M}^{H} \mathrm{r}^{(m)}
$$

f) Obtain $a_{m}$ from $\hat{a}_{\mathrm{m}}$ via slicing.

g) Cancel the interference of $a_{m}$ in $\mathrm{r}^{(m)}$ to obtain the reduced- order problem by

$$
\mathrm{r}^{\left(M^{-1)}\right.}=\mathrm{r}^{\left(M^{\prime}\right)}-\mathrm{h}_{M} a_{M} \quad(20)
$$

h) If $m>1$, let $m=m-1$ and go back to step $\mathrm{b}$. With the corresponding $\left.\mathrm{r}^{\left(m^{-1}\right.}\right), \mathrm{H}^{m^{-1}}, \mathrm{P}^{(\mathrm{M}-1) / 2}$ and $Q_{\alpha}^{M-1}$ instead of $\mathrm{P}^{1 / 2}$ and $\mathrm{Q}_{\alpha}$.

\section{Simulation results}

Simulation is performed using the parameters from Table I. The performance parameters such as BER, SER and PSNR are similar to the results obtained for conventional detection scheme employing MMSE. Figure 6(a) compares the Number of FLOPS required for the conventional Detection scheme employing MMSE and ZF and An Efficient Square-Root Algorithm for BLAST employing MMSE (one complex multiplication and addition requires six and two flops respectively). The efficient square root algorithm outperforms the conventional detection scheme in terms of Number FLOPS required for detecting the received symbols.

Admit of unitary or orthogonal transformation such as householder or givens rotation for detection reduced the computational cost for detection while sustaining the performance obtained with the conventional detection scheme employing MMSE.

Figure 6 (b) (c) compares the time required for detection between conventional detection employing ZF, MMSE and an efficient square root algorithm employing MMSE for $4 \times 4$ and 12x12. Due to the achieved reduction in the Number of floating point operation, reduction in the time required for detection is observed when efficient square root algorithm is employed with MMSE.

\section{An Improved Square Root Algorithm For V-Blast}

The previous algorithm An Efficient Square-Root Algorithm for BLAST algorithm [8] computes the whole nulling matrices $Q_{\alpha}^{m}$ for each deflated sub-channel matrix, while only one column of each is used (i.e., the optimum nulling vector); the intermediate results $P_{M}^{(M-1) / 2}$ computed in the algorithm are discarded without any usage. Thus An Improved Square Root Algorithm [11] for BLAST find's the optimum nulling vectors with the help of $P_{M}^{(M-1) / 2}$, avoiding the computation of $Q_{\alpha}^{m}$. At the same time, the robustness of the improved square-root algorithm is maintained without any inverse or squaring operation. 


\section{Initialization:}

a) Let $m=M$. To Compute an initial $\mathrm{F}=\mathrm{F}$

a1) Set $P_{0}^{1 / 2}=(1 / \sqrt{ } \alpha) I_{M}$

b1) Compute

$$
\begin{gathered}
\Pi_{i}=\left[\begin{array}{cc}
1 & \mathrm{~h}_{i}^{\mathrm{H}} \mathrm{P}_{i-1}^{1 / 2} \\
0_{\mathrm{M}} & \mathrm{P}_{i-1}^{1 / 2}
\end{array}\right] \\
\Pi_{i} \Theta_{i}=\left[\begin{array}{cc}
\mathrm{x} & 0_{\mathrm{M}}^{\mathrm{T}} \\
\mathrm{x} & \mathrm{P}_{i}^{1 / 2}
\end{array}\right]
\end{gathered}
$$

Iteratively for $i=1,2, \cdots, N$.

Where " $x$ " denotes irrelevant entries at this time and $\Theta_{i}$ is any unitary transformations that block lower triangularize the pre-array $\Pi_{i}$

Finally $\mathrm{F}=\mathrm{P}_{N}^{1 / 2}$ is the square root of $\mathrm{P}$ where $\mathrm{P}=\left(\mathrm{H}^{H} \mathrm{H}+\alpha \mathrm{I}\right)^{-1}$

\section{Iterative Detection:}

b) Find the minimum length row of $\mathrm{F}_{\mathrm{m}}$ and permute it to the last row. Permute $\mathrm{H}_{\mathrm{m}}$ accordingly

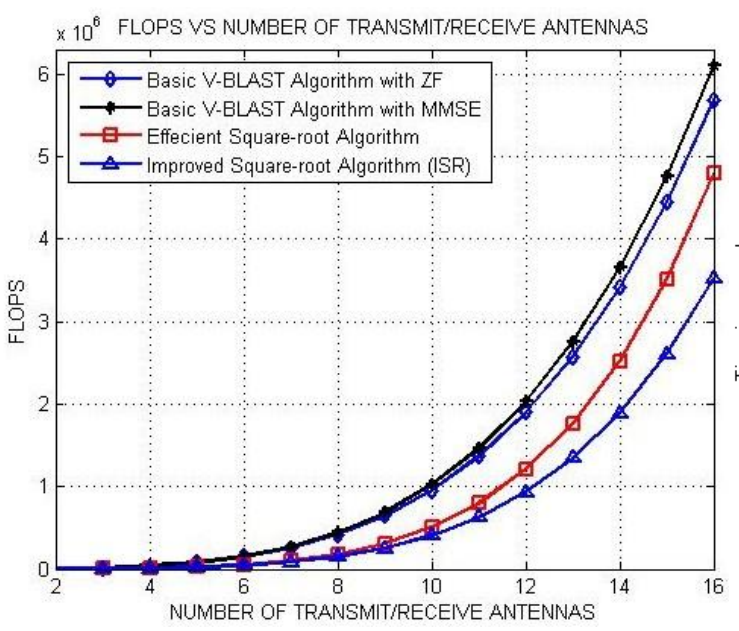

(a)

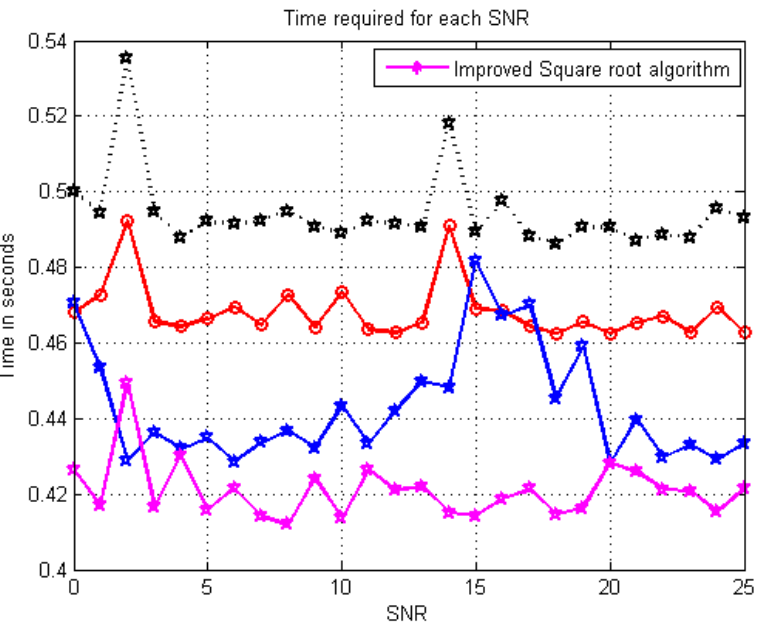

(b)

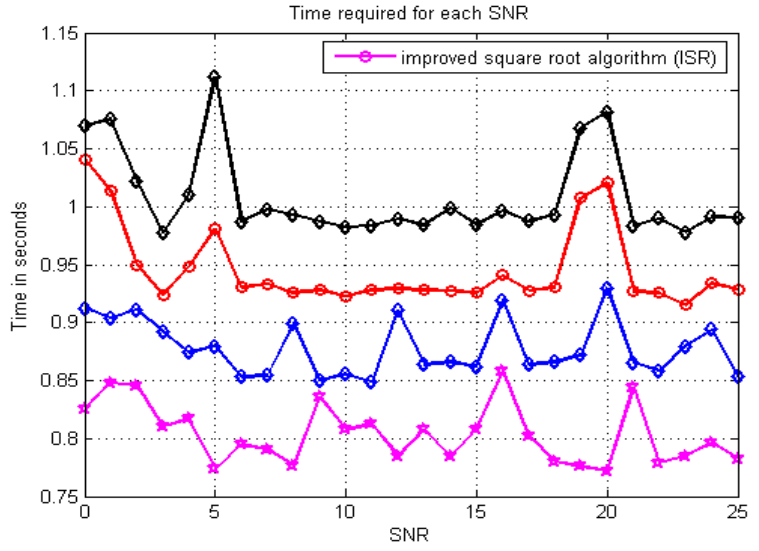

(c) 
Figure 7: (a) TOTAL FLOPS required for conventional detection scheme with ZF and MMSE, an efficient square root algorithm employing MMSE, an improved square root algorithm with MMSE [14] (b) Time required for detection for $4 \times 4$ antenna configuration [14] $\quad$ (c) Time required for detection with $12 \times 12$ antenna configuration (Black: conventional detection with MMSE; red conventional detection with ZF; blue: efficient square root algorithm employing MMSE; magenta: an improved square root algorithm with MMSE)

c) Block upper-triangularize $\mathrm{F}_{\mathrm{m}}$ by

$$
\mathrm{F}_{m} \Sigma=\left[\begin{array}{cc}
\mathrm{F}_{\mathrm{m}-1} & \mathrm{u}_{\mathrm{m}-1} \\
0_{\mathrm{M}}^{\mathrm{T}} & \lambda_{m}
\end{array}\right]
$$

Where $\Sigma$ is a unitary transformation, $\mathrm{u}_{m_{-1}}$ is an $(m-1) \times 1$ column vector, and $\lambda_{m}$ is a scalar.

d) Form the linear MMSE estimate of $a_{m}$,

$$
\left.\widehat{\mathrm{a}}_{\mathrm{m}}=\lambda_{m}\left[\begin{array}{cc}
\mathrm{u}_{\mathrm{m}-1}^{\mathrm{H}} & \left(\lambda_{m}\right)^{*}
\end{array}\right] \mathrm{H}_{\mathrm{m}}^{\mathrm{H}} \mathrm{r}^{(}\right)^{\prime}
$$

e) Obtain $a_{m}$ from $\hat{\mathrm{a}}_{\mathrm{m}}$ via slicing.

f) Cancel the interference of $a_{m}$ in $\mathrm{r}^{(m)}$ to obtain the reduced-order problem by

$$
\mathrm{r}^{\left(M^{-1)}\right.}=\mathrm{r}^{(M)}-\mathrm{h}_{M}
$$

g) If $m>1$, let $m=m-1$ and go back to step P2. With the corresponding $\mathrm{r}^{\left(m^{-1)}\right.}, \mathrm{a}^{m^{-1}}, \mathrm{H}^{m^{-1}}$ and $\mathrm{F}^{m^{-1}}$.

\section{Simulation results}

Simulation is performed using the parameters from Table I. The performance parameters such as BER, SER and PSNR are similar to the results obtained for conventional detection scheme employing MMSE. Figure 7(a) compares the Number of FLOPS required for the conventional Detection scheme employing MMSE and ZF, An Efficient Square-Root Algorithm for BLAST employing MMSE and An Improved Square Root Algorithm for BLAST employing MMSE (one complex multiplication and addition requires six and two flops respectively).since the Improved Square Root Algorithm for BLAST employs unitary transformation and utilizes intermediate results for detection the algorithm outperforms the efficient square root algorithm in terms of Number FLOPS required for detecting the received symbols. From figure7(b),(c) compares the time required for detection for conventional Detection scheme employing MMSE and ZF, An Efficient Square-Root Algorithm for BLAST employing MMSE and An Improved Square Root Algorithm for BLAST employing MMSE with $4 \times 4$ and $12 \times 12$ array. The Time required is directly related to the Number of flops required for execution.

\section{An Improved Square-Root Algorithm For V-BLAST Based On Efficient Inverse Cholesky Factorization}

Further reduction in the number of FLOPS is achieved by employing a fast algorithm for inverse Cholesky factorization used to compute a triangular square-root of the estimation error covariance matrix, it is then applied to propose an improved square-root algorithm for V-BLAST, which speedups several steps in the previous one and can offer further computational savings in MIMO Orthogonal Frequency Division Multiplexing (OFDM) systems. Compared to the conventional inverse Cholesky factorization, the proposed one avoids the back substitution (of the Cholesky factor), and then requires only half divisions. The algorithm is faster than the existing efficient V-BLAST algorithms.[12]

\section{Initialization:}

a) Set $m=M$. Compute $\mathrm{R}_{M}, \mathrm{Z}_{\mathrm{M}}$ and the initial upper triangular $\mathrm{F}=\mathrm{F}_{M}$. This step includes in the sub-steps N1-a, N1-b, N1-c and N1-d.

N1-a) Assume the successive detection order to be $\left[t_{M}, t_{M_{-} 1}, \cdots, t_{1}\right]$. Correspondingly permute $\mathrm{H}$ to be $\mathrm{H}=\mathrm{H}_{M}=$ $\left[\mathrm{h}_{t 1}, \mathrm{~h}_{t 2}, \cdots, \mathrm{h}_{t M}\right]$. 
N1-b) Utilize the permuted $\mathrm{H}$ to compute $\mathrm{R}_{M}$, where we can obtain all $\mathrm{R}_{m_{-1 \mathrm{~s}}}, \mathrm{v}_{m_{-1 \mathrm{~s}}}$ and $\beta_{m_{\mathrm{s}}} \quad$ (for $m=M, \mathrm{M}-1, \cdots$, 2), as shown

$$
\mathrm{R}_{\mathrm{m}}=\left[\begin{array}{ll}
\mathrm{R}_{\mathrm{m}-1} & \mathrm{v}_{\mathrm{m}-1} \\
v_{m-1}^{H} & \beta_{\mathrm{m}}
\end{array}\right]
$$

Where $\mathrm{R}=\mathrm{H}^{H} \mathrm{H}+\alpha \mathrm{I}_{M}$.

N1-c) Compute $F_{1}$ by $F_{1}=\sqrt{R_{1}^{-1}}$ Then use

$$
\begin{array}{r}
\lambda_{m}=1 / \sqrt{\beta_{\mathrm{m}}-\mathrm{v}_{\mathrm{m}-1}^{\mathrm{H}} \mathrm{F}_{\mathrm{m}-1} \mathrm{~F}_{\mathrm{m}-1}^{\mathrm{H}} \mathrm{v}_{\mathrm{m}-1}} \\
\mathrm{u}_{m-1}=-\lambda_{m} \mathrm{~F}_{m-1} \mathrm{~F}_{\mathrm{m}-1}^{\mathrm{H}} \mathrm{v}_{m-1} \text { and } \\
\mathrm{F}_{\mathrm{m}}=\left[\begin{array}{ll}
\mathrm{F}_{\mathrm{m}-1} & \mathrm{u}_{\mathrm{m}-1} \\
0_{\mathrm{M}}^{\mathrm{T}} & \lambda_{m}
\end{array}\right]
\end{array}
$$

To compute $\mathrm{F}_{m}$ from $\mathrm{F}_{m_{-1}}$ iteratively for $m=2,3, \cdots$, to obtain the Initial $\mathrm{F}=\mathrm{F}_{M}$.

N1-d) Compute $\mathrm{Z}_{M}=H_{M}^{H} \mathrm{x}^{\left({ }^{2}\right)}=H_{M}^{H} \mathrm{x}$.
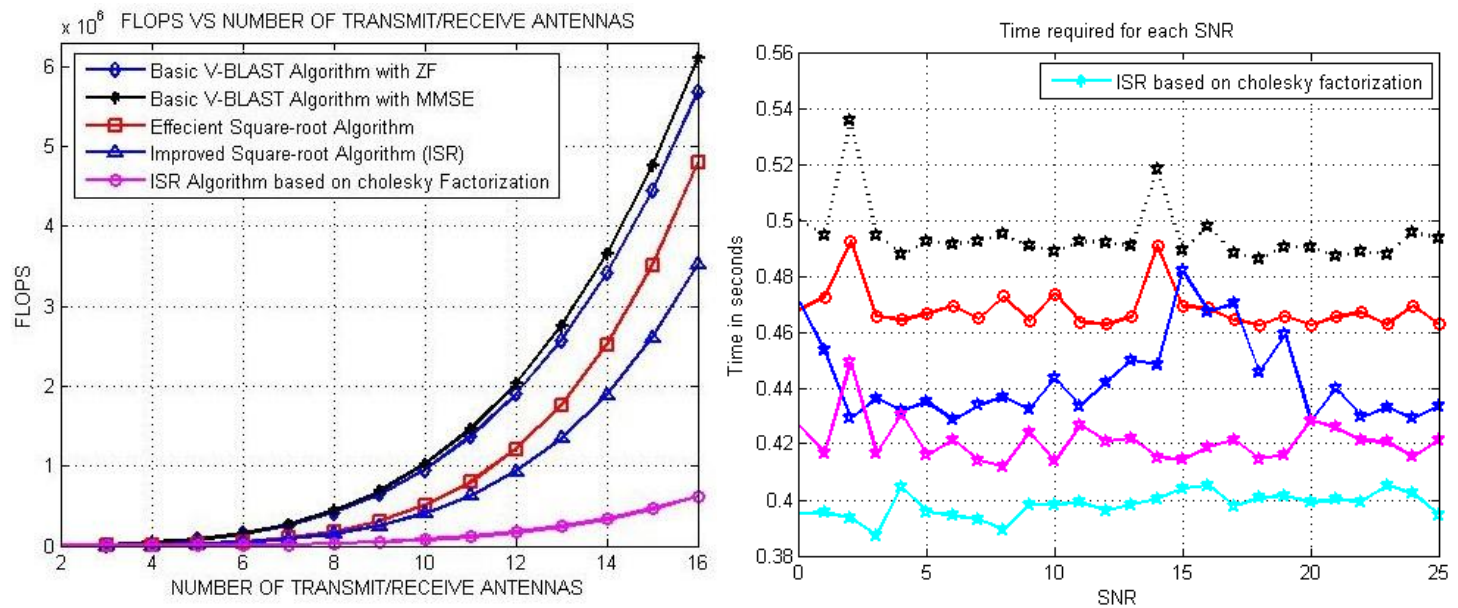

(a) (b)

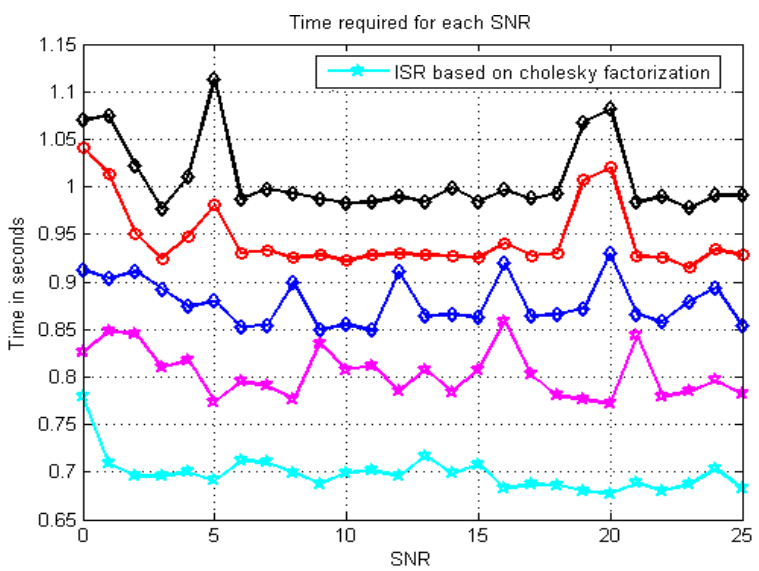

(c) 
Figure 8: TOTAL FLOPS required for conventional detection scheme with ZF and MMSE, an efficient square root algorithm employing MMSE, an improved square root algorithm with MMSE and an improved square root algorithm based on cholesky factorization with MMSE [14]. (b) Time required for detection for 4x4 antenna configuration [14] (c) Time required for detection with $12 \times 12$ antenna configuration (Black: conventional detection with MMSE; red conventional detection with ZF; blue: efficient square root algorithm employing MMSE; magenta: an improved square root algorithm with MMSE and cyan: an improved square root algorithm based on cholesky factorization with MMSE).

\section{Iterative Detection:}

b) Find the minimum length row in $\mathrm{F}_{m}$ and permute it to be the last $m$-th row. Correspondingly permute $\mathrm{Z}_{m}$, and rows and columns in $\mathrm{R}$.

c) Block upper-triangularize $\mathrm{F}_{\mathrm{m}}$ by

$$
\mathrm{F}_{m} \Sigma=\left[\begin{array}{cc}
\mathrm{F}_{\mathrm{m}-1} & \mathrm{u}_{\mathrm{m}-1} \\
0_{\mathrm{M}}^{\mathrm{T}} & \lambda_{m}
\end{array}\right]
$$

Where $\Sigma$ is a unitary transformation, $\mathrm{u}_{m-1}$ is an $(m-1) \times 1$ column vector, and $\lambda_{m}$ is a scalar.

d) Form the least-mean-square estimate $\hat{a}_{m}$ by

$$
\widehat{\mathrm{a}}_{\mathrm{m}}=\lambda_{m}\left[\left(\mathrm{u}_{m-1}\right)\left(\lambda_{m}\right)^{*}\right] \mathrm{Zm}
$$

e) Obtain $a_{m}$ from $\hat{\mathrm{a}}_{\mathrm{m}}$ via slicing

f) Cancel the effect of $a_{m}$ in $\mathrm{Zm}$ by

$$
\mathrm{Z} m-1=z_{m}^{[-1]}-a_{m} \mathrm{~V} m-1
$$

Where $\mathrm{z}_{\mathrm{m}}^{[-1]}$ is the permuted $\mathrm{z} m$ with the last entry removed, and $\mathrm{v} m-1_{-1}$ is in the permuted $\mathrm{R}_{m}$

g) If $m>1$, let $m=m-1$ and go back to step $\mathrm{N} 2$ with the corresponding $\mathrm{Z}_{m-1}, \mathrm{a}_{m-1}, \mathrm{R}_{m_{-1}}$ and $\mathrm{F}_{m_{-1}}$.

\section{Simulation results}

Simulation is performed using the parameters from Table I. The performance parameters such as BER, SER and PSNR are similar to the results obtained for conventional detection scheme employing MMSE.

Figure 8 (a) compares the Number of FLOPS required for the conventional Detection scheme employing MMSE and ZF, An Efficient Square-Root Algorithm for V-BLAST employing MMSE, An Improved Square Root Algorithm for V-BLAST employing MMSE and An Improved Square-Root Algorithm for V-BLAST Based on Efficient Inverse Cholesky Factorization employing MMSE (one complex multiplication and addition require six and two flops respectively). An Improved Square-Root Algorithm for VBLAST Based on Efficient Inverse Cholesky Factorization outperforms all the above mentioned Algorithms and is faster than the existing efficient V-BLAST algorithms.

Figure 8 (b),(c) compares the time required for detection Due to the achieved reduction in the Number of floating point operation, reduction in the time required for detection is observed when Improved Square-Root Algorithm for V-BLAST Based on Efficient Inverse Cholesky Factorization is employed with MMSE.

\section{CONCLUSION}

This paper provides a detailed comparison of various detection schemes employed in V-BLAST systems with modulation schemes such as M-QAM, M-PAM, M-PSK for 4x4 and 12x12 array. Parameters considered include BER, SER, PSNR and FLOPS. Simulation results show optimum performance for VBLAST is achieved with M-QAM modulation scheme, whereas MMSE outperforms ZF in terms of BER SER and PSNR at the cost of increase in number of FLOPS. The number of FLOPS required for MMSE at 16 transmitting and 16 receiving antennas is $6.2 \times 10^{6}$, FLOPS of $5.8 \times 10^{6}$ for ZF is observed. Reduction in the number of FLOPS required for detection is accomplished by efficient square root algorithm with MMSE which employs unitary transformation to avoid squaring and matrix inversion operation, the number of FLOPS required with 16 transmitting and 16 receiving antennas is $4.8 \times 10^{6}$, A reduction of $1 \times 10^{6}$ FLOPS and $1.4 \times 10^{6}$ FLOPS is achieved compared to conventional detection scheme employing ZF and MMSE respectively. Reduction in the number of FLOPS compared to efficient square root algorithm was accomplished by the improved square root algorithm which utilizes intermediate results that were discarded without any usage in the 
efficient square root algorithm. The number of FLOPS required for detection with improved square root algorithm employing MMSE for 16 transmitting and 16 receiving antennas is $3.5 \times 10^{6}$, a reduction of $1.3 \times 10^{6}$ FLOPS is achieved compared to the efficient square root algorithm with MMSE and reduction $2.3 \times 10^{6}, 2.7 \times 10^{6}$ FLOPS is achieved compared to conventional detection scheme employing ZF, with MMSE respectively. Further reduction in number of FLOPS is achieved by employing a fast algorithm to compute a triangular square root of the estimation error covariance matrix. The number of FLOPS required improved square root algorithm based on efficient inverse cholesky factorization with MMSE for 16 transmitting and 16 receiving antennas is $0.6 \times 10^{6}$, a reduction of $2.9 \times 10^{6}, 4.2 \times 10^{6}, 5.2 \times 10^{6}$ and $5.6 \times 10^{6}$ is achieved compared to improved square-root algorithm, the efficient square root algorithm and the conventional detection scheme employing ZF,MMSE respectively. The algorithm is faster than the existing efficient V-BLAST algorithms.

\section{REFERENCES}

[1] J. H. Winters, "On the capacity of radio communication systems with diversity in a Rayleigh fading environment," IEEE Journal on Select Areas in Communications, June 1987, Issue: 5, vol. 5, pp. 871-878.

[2] G.J. Foschini and M.J. Gans, "On limits of wireless communications in a fading environment when using multiple antennas", Wireless Personal Communications, Mar 1998, Issue 3, vol. 6, pp. 311-335.

[3] T. L. Marzetta and B. M. Hochwald, "Capacity of a mobile multiple-antenna communication link in Rayleigh flat fading," IEEE Transactions on Information Theory, Jan. 1999, Issue 1, vol. 45, pp. 139-157.

[4] G. G. Raleigh and J. M. Cioffi, "Spatio-temporal coding for wireless communication," IEEE Transactions on Communications, Mar.1998, Issue 3, vol. 46, pp. 357-366.

[5] G.J. Foschini, "Layered space-time architecture for wireless communication in a fading environment when using multi-element antennas" Bell Labs Technical Journal, Aug 2002, issue 3, vol. 1, pp.41-59.

[6] P. W. Wolniansky, G. J. Foschini, G. D. Golden, and R. A. Valenzuela, "V-BLAST: an architecture for realizing very high data rates over the rich scattering wireless channel ", URSI International Symposium on Signals, Systems and Electronics, Pisa, Oct 1998, DOI: 10.1109/ISSSE.1998.738086, pp - 295 - 300.

[7] Shankar.P , Dr. S.A Hariprasad "Analysis of Efficient square root algorithm for V-blast MIMO Wireless Communications “,International Journal of Engineering Research and Applications (IJERA) Vol. 2, Issue4, JulyAugust 2012, pp.2153-2161.

[8] B. Hassibi, "An efficient square-root algorithm for BLAST," IEEE International Conference in Acoustics, Speech, and Signal Processing, (ICASSP '00), June 2000, DOI: 10.1109/ICASSP.2000.859065, vol 2, pp. 737-740.

[9] G. H. Golub and C. F. Van Loan, Matrix Computations, Johns Hopkins University Press, 3rd edition, 1996, ISBN 0-8018-5414-8.

[10] T.kailath,A.H sayed and B hassibi . "Linear Estimation", Prentice-hall, $1^{\text {st }}$ edition, April 2000,ISBN: 0-13-0224642.

[11] H. Zhu, Z. Lei, and F. P. S. Chin, "An improved square-root algorithm for BLAST", IEEE Signal Processing Letters, Sept. 2004, issue 9, vol.9, pp-772 - 775 .

[12] Hufei Zhu, Wen Chen, Bin Li, and Feifei Gao, "An Improved Square-Root Algorithm for V-BLAST Based on Efficient Inverse Cholesky Factorization" IEEE transactions on wireless communications, jan 2011,issue 1, vol. 10, pp- $43-48$.

[13] Shankar.P, Dr. S.A Hariprasad "Analysis of Efficient square root algorithm for V-blast MIMO Wireless Communications" International Journal of Engineering Research and Applications, August 2012, Issue 4, Vol. 2, pp.2153-2161.

[14] Shankar.P, Dr. S.A Hariprasad " Analysis of Improved Square-Root Algorithm Based on Efficient Inverse Cholesky Factorization for V-blast MIMO Wireless Communications " International Journal of Computer Networks and Wireless Communications, August 2012, issue 4, Vol.2, pp 465-480.

[15] Shankar.P, Dr. S.A Hariprasad "Performance Analysis of M-ary Digital modulation techniques with ZF and MMSE equalizers for V-Blast MIMO wireless communication ", $2^{\text {nd }}$ world congress on information and communication technologies (communicated).

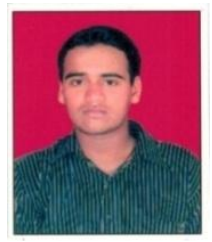

Shankar.P received B.E in electronics and Communication from Visvesvaraya Technological University (VTU) in the year 2010, and currently pursuing M.TECH in Communication System from R.V.C.E Bangalore, Karnataka, India. His main research interests include antennas and propagation for personal communications, MIMO, radar remote sensing, numerical electro-magnetic, and optical fiber communications.

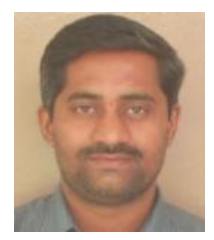

Dr Hariprasad S.A obtained his $\mathrm{PhD}$ from Avinashilingam University for Women, Coimbatore in the area of digital controller's .He is having teaching experience of 22 years and five years of research experience. He has published 35 papers in international national journals and conferences. He has also published a text book on advanced Microprocessor and reviewed books on Microwave engineering and won best teacher award (twice) from RSST and appreciation award from ISTE. His research areas of interest are embedded systems and RF systems. 